\title{
Contribuindo para a discussão do Qualis de terapia ocupacional no Brasil ${ }^{*}$
}

\section{Contributing for the discussion of the Qualis classification in occupational therapy in Brazil}

\author{
Denise Dias Barros ${ }^{(1)}$, Fátima Corrêa Oliver $^{(2)}$
}

BARROS, D. D., OLIVER, F. C. Contribuição para a discussão do Qualis de terapia ocupacional no Brasil. Rev. Ter. Ocup. Univ. São Paulo, v. 14, n. 2, p. 52-63, maio/ago. 2003.

RESUMO: Objetivo. Caracterizar a produção bibliográfica de pesquisadores terapeutas ocupacionais pertencentes a grupos de pesquisa coordenados por terapeutas ocupacionais, credenciados junto ao $\mathrm{CNPq}$ (Diretório de Grupos de Pesquisa, versão 4.1). Estes representam a maior parte daqueles diretamente envolvidos em atividades de pesquisa no país. Metodologia. Foram considerados os artigos completos publicados em periódicos nacionais $\mathrm{e}$ internacionais de terapcutas ocupacionais com mestrado e/ou doutorado, até o ano de 2000 . levantados a partir da base de dados de currículos no $\mathrm{CNPq}$. A produção foi caracterizada segundo periódicos (indexados e não indexados em bases de dados bibliográficas), áreas de conhecimento (biológico, saúde, educação c ciências sociais) e instituições de origem de autores. Resultados. Foram localizados 169 artigos de 46 autores, no período de 1985 a 2000 , divulgados em 54 periódicos. A produçāo nacional representou $85,8 \%$ dos 169 artigos divulgados. A realização de mestrados e doutorados por tcrapcutas ocupacionais repercutiu na produçāo bibliográfica apenas na década de 90 . Nesse campo emergente que é a terapia ocupacional ficaram evidenciados: concentração dos autores na região Sudcste, sobretudo no Estado de São Paulo, intensa dispersāo da produção em diferentes periódicos, número restrito de autores $\mathrm{c}$ pequena produção $\mathrm{cm}$ números absolutos. Perceberam-se os limites de uma avaliação da produção docente que supervaloriza a produção indexada em bases de dados bibliográficas de língua inglesa. Ficou evidente a necessidade do estabelecimento de estratégias mais coletivas para aumentar em números absolutos a produção bibliográfica, tanto de artigos como de livros, evitar a dispersão da produção em diferentes veículos e fomentar a discussão do tema entre os pesquisadores $\mathrm{e}$ docentes das Universidades.

DESCRITORES: Terapia ocupacional/educação. Educação de pós-graduação. Pesquisa. Estratégias.

\footnotetext{
(1) Prolessora Doutora do Departamento de Fisioterapia, Fonoaudiologia e Terapia Ocupacional da Faculdade de Medicina da Universidade de São Paulo, Doutora em Sociologia pela USP.

(2) Doutora em Saúde Pública. Docente do Curso de Terapia Ocupacional do Departamento de Fisioterapia, Fonoaudiologia e Terapia Ocupacional da USP.

Endereço para correspondência: Departamento de Fisioterapia, Fonoaudiologia e Terapia Ocupacional da Faculdade de Medicina da Universidade de São Paulo. Rua Cipolânea, 51.05360-000. São Paulo, SP. e-mail: fcoliver@usp.br
} 


\section{A terapia ocupacional vem se constituindo como área de ensino e pesquisa nas universidades} brasileiras a partir de 1980. Nesta década, definiu-se a ampliação do ensino para 4 anos de graduação pelo Ministério de Educação e Cultura, que serviu de base para a reestruturação curricular do ensino de graduação nas principais instituições de ensino superior existentes (LOPES, 1991, p. 63).

É desse período o início da formação de pesquisadores através do ingresso de terapeutas ocupacionais em programas de pós-graduação em diferentes áreas do conhecimento, exigência da cárreira docente, principalmente nas universidades públicas.

Até a década de 90 , a profissāo estava voltada para a sua incorporação no mercado de trabalho, sobretudo, na área da saúde. O ritmo lento da formação de pesquisadores terapeutas ocupacionais deveu-se, em parte, à inexistência de pós-graduação em terapia ocupacional no país, fato que levou os profissionais a dedicarem grande parte do tempo à compatibilizaçũo de seus currículos àqueles exigidos para o desenvolvimento dos programas de mestrado e doutorado em áreas afins.

Em 1986, havia nas escolas públicas apenas dois mestres e um doutor, enquanto que nas escolas particulares não havia docentes com pós-graduação strictu sensu. Dez anos depois esse quadro alterou-se para 26 mestres e 7 doutores nas universidades públicas c 3 mestres em escolas particulares (EMMEL; LANCMAN, 1998, p. 33). Outra mudança relevante ocorreu no final dos anos 90 , devido à conclusão de grande parte dos doutorados, confirmando a projeção realizada por Emmel e Lancman (1998, p. 37).

Atualmente há 35 cursos de graduação em terapia ocupacional no país, com expressivo aumento do número de instituições privadas, que de 10, em 1998, passaram para 26, no ano 2002. O mesmo crescimento não ocorreu com o ensino público que passou de 7 para 9 instituições. Se o desenvolvimento de atividades de pesquisa tem sérias dificuldades nas instituiçōes de ensino superior federais e estaduais do país, elas são praticamente estranhas às exigências contratuais das instituiçōes privadas. Raras são as que possuem carreiras acadêmicas vinculadas à pesquisa e a maioria contrata professores com base na carya horária didática e/ou administrativa. Atualmente, são cerca de 1985 vagas oferecidas pelo menos uma vez ao ano em todo o país. Destas, 285 são de instituições públicas ${ }^{(1)}$. Por esses motivos podem-se levantar as seguintes questōes fundamentais para o ensino e pesquisa na terapia ocupacional: Qual é o perfil da produção bibliográfica? Que impacto tem tido as exigências da CAPES para a criaçāo de programas de pós na área? Sob que parâmetros constituir um sistema de classificação da produção acadêmica? Qual o lugar da pesquisa no futuro da terapia ocupacional? São muitas as questōes e os fóruns de discussão permanecem insuficientes. São propostas deste estudo caracterizar a produção de artigos publicados em periódicos científicos por terapeutas ocupacionais no país e contribuir para a reflexão sobre os sistemas de avaliação da produçĩo acadêmica, a pós graduação strictu sensu e as possibilidades para o desenvolvimento da pesquisa.

\section{Procedimentos}

O levantamento e as análises dos dados deste estudo foram realizados em duas etapas. Na primeira, obtivemos informações que permitiram: a) identificar os terapeutas ocupacionais cadastrados nos grupos de pesquisa coordenados por terapeutas ocupacionais informações obtidas no Diretório de Grupos de Pesquisa, versão 4.1 do $\mathrm{CNPq}$.; b) localizar no Currículo Lattes os artigos completos, publicados em periódicos a partir dos nomes dos pesquisadores encontrados; c) conhecer as bases de indexação bibliográficas dos artigos encontrados no período de 1985 a 2000; d) levantar os critérios da CAPES para criação de novos programas de estudos pós-graduados.

Na segunda etapa foi: a) caracterizada a produção divulgada em periódicos por terapeutas ocupacionais, discutindo os critérios de avaliação da produção bibliográfica dos pesquisadores ligados a universidades; b) caracterizada al distribuição de artigos publicados por terapeutas ocupacionais mestres e doutores, vinculados a grupos de pesquisa coordenados por terapeutas ocupacionais, segundo: periódicos (indexados e não indexados em bases de

\footnotetext{
"1) Informaçōes obtidas do cadastro das instituiçōes de ensino superior do Ministério de Educação, realizado pelo Instituto Nacional de Estudos e Pescluisas Educacionais (INEP). http://www.educacaosuperior.inep.gov.br/ e junto à UNESP http://www.marilia.uncsp.br/ensino/graduacao/ index.htm, ambos no dia 27 de junho de 2003.
} 
dados bibliográficos), áreas de conhecimento (biológico, saúde, educação e ciências sociais) $\mathrm{e}$ instituições de origem dos autores; c) analisada a produção e discutida a necessidade de criação de pós-graduação.

\section{Pesquisadores em terapia ocupacional}

Nos 16 grupos de pesquisa coordenados por terapeutas ocupacionais identificados no diretório $\mathrm{CNPq}$ 4.1 de 2000 , foram encontrados 27 doutores e 19 mestres, sendo que os doutores pertencem a várias universidades no Brasil. Alguns grupos possuem pesquisadores de mais de uma instituição e há pessoas participando de até três diferentes grupos. Como a pesquisa está intrinsecamente vinculada aos estudos pós-graduados, consideramos incontornável retomar informaçōes sobre a capacitação dos 46 profissionais que compõem este estudo. São deles igualmente a produção que será considerada posteriormente.

Os primeiros mestrados foram concluídos principalmentc por docentes vinculados a universidades públicas, particularmente da Universidade de São Paulo e da Universidade Federal de São Carlos, demonstrando investimento institucional na indissociabilidade das atividades de ensino e pesquisa. Por meio da atualização dos dados curriculares dos pesquisadores, pudemos constatar que houve uma evolução da titulação nos 16 grupos identificados. A evolução de mestrados, doutorados e pós-doutorados pode ser observada no Gráfico 1 . Havia em 2002, 32 doutores, 20 mestres e 4 pós-doutores concluídos ( 3 na França e 1 no Canadá).

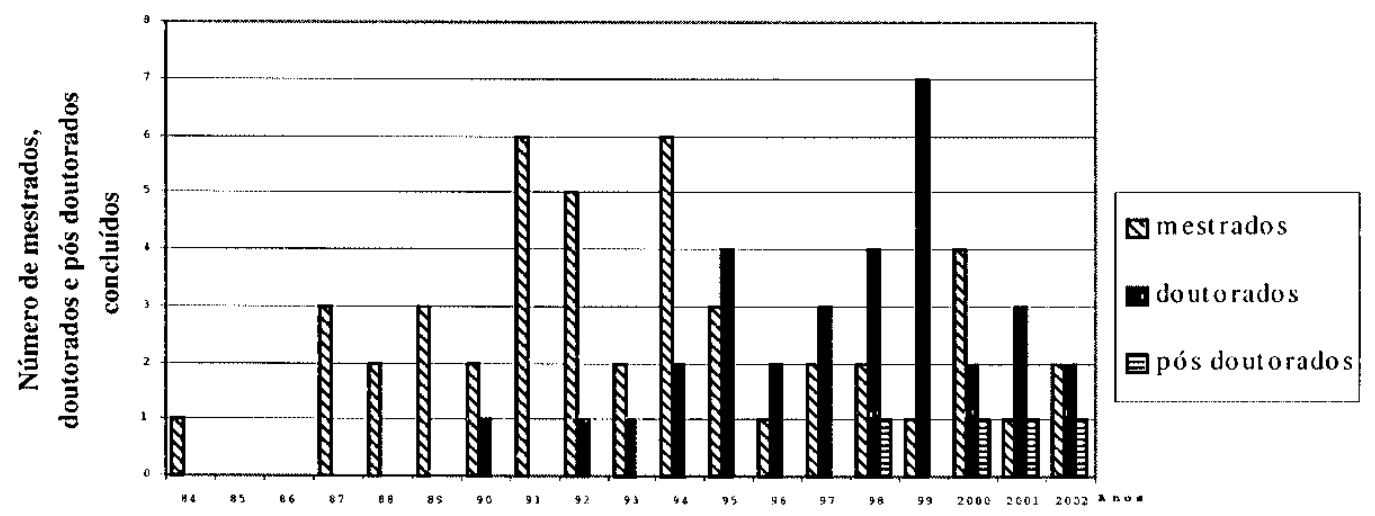

Gráfico 1. Anos de conclusão de mestrados, doutorados e pós doutorados de terapeutas ocupacionais dos grupos de pesquisa CNPq, Diretório versão 4.1 - 2000.

Cabe ressaltar que além dos desafios inerentes à formação docente, os terapeutas ocupacionais brasileiros enfrentam outros problemas. Alguns são comuns a outras áreas como a forte carga de trabalhos burocrático-administrativos (MAGALHÃES, 1998, p. 120) e a falta de recursos humanos e financeiros sendo muitas vezes chamados a captar recursos para pesquisa e até para o funcionamento administrativo e organizacional. Outros, são dificuldades específicas ligadas à inserção institucional da terapia ocupacional, pois muitas graduações não se constituem nem mesmo como departamentos próprios, o que restringe a autonomia de decisões frente a questões de ensino e a definiçāo de diretrizes para a pesquisa em terapia ocupacional.

A realização de mestrados e doutorados por terapeutas ocupacionais repercutiu na produção bibliográfica apenas na década de 90 . O processo de capacitação docente parece ter sido o principal fator para o expressivo crescimento de publicaçōes em periódicos de autoria de terapeutas ocupacionais.

\section{Caracterização dos artigos publicados por} pesquisadores terapeutas ocupacionais

No Quadro 1, ficaram demonstrados os nomes dos 16 grupos de pesquisa, sua composição e distribuição por instituição de ensino. Estes grupos contavam com a presença de mestres, mestrandos, doutores e doutorandos além de graduados e especialistas. Nele fica evidente a concentração dos grupos de pesquisas nas universidades públicas - 13 grupos - onde predominam doutores e doutorandos. 
Rev. Ter. Ocup. Univ. São Paulo, v. 14, n. 2, p. 52-63, maio/ago. 2003.

Quadro 1. Composição dos grupos de pesquisa com área predominante Terapia Ocupacional, no Diretório CNPq, coordenados por terapeutas ocupacionais, segundo capacitação registrada nos censos de 2000 e $2002^{*}$.

\begin{tabular}{|c|c|c|c|c|c|c|c|c|c|c|c|c|}
\hline \multirow[t]{2}{*}{ Nome do Grupo m Instituição } & \multicolumn{6}{|c|}{ Censo 2000} & \multicolumn{6}{|c|}{ Censo 2002} \\
\hline & $\mathbf{D}$ & Dndo & $\mathbf{M}$ & Mndo & Grad./Esp. & Total & D & Dndo & $\mathbf{M}$ & Inodo & Grad/Esp. & Total \\
\hline \multicolumn{13}{|l|}{ Universidade de São Pauto - USP } \\
\hline 1. Políticas. Ações sociais, Cultura e Reabilitaçāo $\oslash$ & 6 & 3 & 1 & - & - & 10 & 8 & 1 & 1 & - & - & 10 \\
\hline 2. Grupo de estudo de Trabalho. Saúde c Terapia Ocupacional $\varnothing$ & 4 & - & 2 & - & -11 & 7 & 6 & $-f ?$ & 1 & - & - & 7 \\
\hline $\begin{array}{l}\text { 3. Laboratorio de Estudo e Pesquisa Arte e Corpo } \mathrm{cm} \\
\text { Terapia Ocupacional }\end{array}$ & - & 4 & - & - & $4 /$. & 8 & 1 & 1 & - & 1 & $-/ 1 ?$ & 4 \\
\hline $\begin{array}{l}\text { 4. Atividade Motora bumana em integração com processos } \\
\text { psicossociais }\end{array}$ & 1 & - & - & 2 & - & 3 & $\mathbf{I}$ & - & - & 1 & . & 3 \\
\hline $\begin{array}{l}\text { 5. Estudos Epistemológicos e Clínicos de Terapia } \\
\text { Ocupaciona1*Ø }\end{array}$ & 1 & - & - & - & 1 & 2 & - & - & . & - & $\cdot$ & . \\
\hline $\begin{array}{l}\text { 6. REATA - Liboratório de Esstulos en Reabilitaçio e } \\
\text { Tecnologia Assistiva }\end{array}$ & 2 & 1 & 1 & - & $1 / 2$ & 7 & 3 & I & 1 & $\cdot$ & $1 / 2$ & 7 \\
\hline Laboratório de Estudos sobre Deficiência e Cotidiano \#\# & - & - & . & - & - & - & 2 & - & - & 1 & 1 & 4 \\
\hline \multicolumn{13}{|l|}{ Universidade Federal de São Carlos - UFSCar } \\
\hline 7. Terapia Ocupacional no campo social $\varnothing$ & 5 & - & . & 2 & $\%$ & 8 & 4 & - & - & 2 & -11 & 7 \\
\hline 8.Formaçăo e Capacilaç̧io em Terapia Ocupacional $\varnothing$ & 6 & 1 & - & - & - & & 6 & . & . & - & - & 6 \\
\hline 9.Terapia Ocupacional na Infâncial e Adolescêuciał** & 3 & 1 & - & - & -12 & 7 & 4 & - & - & - & - & 4 \\
\hline 10. Terapia Ocupacionad e Saúde Mental ver $\varnothing$ & 3 & 2 & - & 1 & - & 6 & 4 & . & - & - & - & 4 \\
\hline 11. Grupo de Pesquisa em Disfunçīo Física e Saúde ver $\varnothing$ & 4 & 1 & - & 1 & - & 6 & 4 & I & 1 & - & . & 5 \\
\hline \multicolumn{13}{|l|}{ Universidade Federal de Mlinas Gerais - UFMG } \\
\hline 12. Satúde Física e Reabilitaçăo & - & 2 & 1 & - & $-n$ & 4 & - & 2 & 2 & - & . & 3 \\
\hline 13. Avaliaļato do Desenvolvimento e Deseinpenho Infantil ver 8 & 3 & 1 & - & - & -11 & 5 & 3 & 1 & 1 & - & $! /$ & 5 \\
\hline \multicolumn{13}{|l|}{ Pontifícia universidade Católica de Campinas - PLCC } \\
\hline $\begin{array}{l}\text { 14. Políticas, Fundamentos, Formaçâo e Açōes em Terapia } \\
\text { Ocupacional ver } D\end{array}$ & 3 & 1 & - & 6 & -12 & 12 & 5 & - & 3 & - & - & 8 \\
\hline \multicolumn{13}{|l|}{ Universidade de Sorocaba -- UNISC, } \\
\hline 15. Grupo de Pesquisa em Terapia Ocupaciona1*** & 1 & - & 4 & 3 & $2 \%$ & 10 & $\cdot$ & - & - & - & - & - \\
\hline \multicolumn{13}{|l|}{ Universidade Potiguar - UNP } \\
\hline 16. Terapia Ocupacional e Açĩo Comuniltiria*** & - & - & - & - & $2 /$ & 2 & $\cdot$ & - & - & - & - & . \\
\hline
\end{tabular}

\# Existem pesquisiddores em mais de un gnpo; * a docente responsável pelo grupo desligou-se da Universidade em 2002; ** no Censo de 2002, fouve alteraçäo do nome do grupo para Terapia Ocupacional: Atividade, Desenvolvimento e Qualidade de Vida: *** grupos que não constam no censo de 2002 ; \#\# Gnpo criado em 2002; $\varnothing$ Grupo com pesquisador com pós-doutorados.

Na Tabela 1, ficaram demonstrados os 169 artigos dos 46 terapeutas ocupacionais (mestres e doutores), escritos indiviaualmente ou em conjunto, no período de 1985 a 2000 e observou-se distribuição crescente dos artigos, sobretudo a partir de 1996. Entre 1992 e 1995 foram publicados 21 artigos e a variação do número de publicaçōes se manteve entre 2 e 8 artigos anuais, o que pode ser explicado por ser esse o período de inserção de grande parte desses pesquisadores em programas de doutorado. Entre 1996 e 1999 foram publicados 79 artigos, representando um aumento significativo, com 16 a 23 artigos anuais, sendo que o aumento se manteve em 2000 , com 39 publicaçōes. Outra observação relevante foi a da dispersão dos artigos em 
grande número de periódicos, 41 nacionais e 12 estrangeiros. $\mathrm{Na}$ Tabela 2 foram apresentados apenas os artigos publicados em revistas nacionais indexadas em bases nacionais e/ou internacionais.

Tabela 1. Distribuição dos artigos nacionais e internacionais, segundo ano de publicação, no período de 1985 a 2000.

\begin{tabular}{|c|c|c|c|c|c|c|c|c|c|c|c|c|c|c|c|c|c|}
\hline Ano & 85 & 86 & 87 & 88 & 89 & 90 & 91 & 92 & 93 & 94 & 95 & 96 & 97 & 98 & 99 & 2000 & Total \\
\hline Nacional & 1 & 1 & - & 3 & 1 & 7 & 14 & 4 & 8 & 1 & 4 & 13 & 18 & 20 & 16 & 34 & 145 \\
\hline Internacional & - & - & - & - & 1 & - & 2 & 1 & - & I & 2 & 3 & 1 & 3 & 5 & 5 & 24 \\
\hline Total & 1 & 1 & - & 3 & 2 & 7 & 16 & 5 & 8 & 2 & 6 & 16 & 19 & 23 & 21 & 39 & 169 \\
\hline
\end{tabular}

Tabela 2. Artigos de autores únicos e coletivos publicados até $2000 \mathrm{em}$ periódicos nacionais indexados, segundo instituições e indexadores.

\begin{tabular}{|c|c|c|c|c|c|c|c|c|}
\hline \multirow{3}{*}{ Periódicos nacionais } & \multicolumn{6}{|c|}{ Artigos publicados até 2000} & \multirow{3}{*}{ Total } & \multirow{3}{*}{ Indexadores } \\
\hline & \multirow{2}{*}{$\begin{array}{l}\text { De } \\
\text { autores } \\
\text { coletivos }\end{array}$} & \multicolumn{5}{|c|}{ De autor ínico } & & \\
\hline & & USP & UFSCar & UFMG & PUCCamp & UNISO & & \\
\hline Revista de Terapia Ocupacionial da USP/SP & 15 & 27 & 4 & 1 & 1 & 1 & 49 & l.ilacs \\
\hline Temas sobre desenvolvinkentosP & 10 & - & - & 1 & - & - & 11 & Lilacs \\
\hline Jornal Brasileiro de Psiquiatria/RJ. & 2 & - & 1 & - & . & - & 3 & $\begin{array}{l}\text { Embase, Litacs, Biol-Abstr; Medline; Psychol- } \\
\text { Abstr; Psycinfo; E-P'syche }\end{array}$ \\
\hline Revista Quaestio/Sorocaba & 2 & - & 1 & & & & 3 & Edubase \\
\hline Revista de Ciências Médicas/PUCCamp & 1 & - & & & 1 & & 2 & Lilites \\
\hline A Folha Médica/SP & 1 & . & & & & & 1 & Embase, Lilacs, Biol-Abstr, Embase; Medline \\
\hline Cadernors de Siúde Pública - Iriocruz/RJ & -1 & - & - & - & - & 1 & & $\begin{array}{l}\text { Lilacs, Medline, SciElo, Sociological Abstracts, } \\
\text { Sociat Ptanning/Policy \& Development, } \\
\text { Abstract on Higiene and Communicable } \\
\text { Diseases, Cab Abstracts }\end{array}$ \\
\hline Educaçăto e Socicdade: Revista & 1 & - & - & - & + & . & 1 & $\begin{array}{l}\text { Cont-Py-Educ; lbr, Contents, Sociological } \\
\text { Abstract, Scielo, Lang-and-Litng-Behay-Abstr }\end{array}$ \\
\hline Hansenologia Internationalis /SP & 1 & - & + & - & - & - & 1 & $\begin{array}{l}\text { Lilacs, Biol-Abstr, Embase, Medline. Trop-Dis- } \\
\text { Bull }\end{array}$ \\
\hline Puidćia: Ciadenoos de Psicologia e Educação & - & - & 1 & - & - & - & 1 & Lilacs \\
\hline Pediatria/SP & 1 & & - & - & - & - & 1 & Lilacs, Biol-Ahstr, Embase. Medline \\
\hline Psicanálise e Universidade/SP. & -1 & - & - & - & - & 1 & & Lililes, Psi-periódicos \\
\hline Psicologia: Teoria e Pesquisi/l3risalia & l & & - & - & - & - & 1 & $\begin{array}{l}\text { Lilacs, Scielo, Psycinfo, Psychol-abstr; E- } \\
\text { Psyche }\end{array}$ \\
\hline Revista Brasileira de Estudos Pedagógicos & 1 & - & + & • & $\cdot$ & - & 1 & $\begin{array}{l}\text { Jlisp-Amer-Per-Int;; Sociol-Abstr; Soc-Serv- } \\
\text { Abstr, Lang-And-Lang-Behav-Abstr }\end{array}$ \\
\hline Revista Brasileira de Medicina/RJ & 1 & - & - & . & - & - & 1 & $\begin{array}{l}\text { Lilacs, Embase, Isi, Biol-Abstr. Curr-Cont; } \\
\text { Abstr-Hyg, Trop-Dis-Bull. Inpharma, Weed- } \\
\text { Abstr; Bairy-Sci-Abstr, Rev-Arom-And-Med- } \\
\text { Plants }\end{array}$ \\
\hline Revista Brasikira de Neurologia/RJ & 1 & $\cdot$ & - & - & - & - & 1 & $\begin{array}{l}\text { Lilacs, Embase, Psycinfo; Embase; Medine; E- } \\
\text { Psyche }\end{array}$ \\
\hline $\begin{array}{l}\text { Revista dia Asseciaçüo Brasileira de } \\
\text { Psiquiatria/Sp }\end{array}$ & 1 & * & - & - & + & - & 1 & $\begin{array}{l}\text { Lilacs, Scielo, Psycinfs, Biol-Abstr; Embase, } \\
\text { Medline; P'syclool-Abstr; I'sycinfo; E-Psyche }\end{array}$ \\
\hline Revista de Psicologia da USi's/P' & - & - & 1 & - & - & - & 1 & Lilacs, SciELO \\
\hline Revista de Saride PúblicidSP. & I & - & - & - & . & - & I & $\begin{array}{l}\text { Lmbase, iSI, Lilacs, Medline, SciblO, Biol- } \\
\text { Abstr: Curr-Cont, Nutr-Abstr; Ssci, Asca, C-I- } \\
\text { Abstr; Dent-Ind, Trop-Dis-Bull, Environ- } \\
\text { Sci-Poll-Mgmt, Virol-Abstr, SeI-Water-Res- } \\
\text { Abstr, Wild-Rev; Dairy-Sci-Abstr, Risk-Abstr, } \\
\text { Sugar-Ind-Abstr; Rural-Devel-Abstr, Rurat- } \\
\text { Recreat-Tour-Abstr }\end{array}$ \\
\hline Outros * & 5 & - & - & - & - & - & 5 & liflacs \\
\hline TOTAL & 45 & 29 & 8 & 2 & 2 & 1 & 87 & \\
\hline
\end{tabular}

* Um artigo em cada um dos seguintes periódicos: Revista Distúrbios da Comunicação/SP; Revisla Latinoamericama de P'sicopatologia liunu/SP; Revisti Paulista de Pediatria/SP; Revista Brasileira de Fisioterapia/S.Carlos e Revistil de Fissioterapia da USP/SP.

As bases para classificaçāo de periódicos que foram criadas para reunir e favorecer a circulação das informações científicas, tornaram-sc incontornáveis como referência para avaliar a produção das diferentes áreas do conhecimento. Algumas delas adquiriram mais prestígio devido a sua utilização pela CAPES, CNPq e 
Rev. Ter. Ocup. Univ. São Paulo, v. 14, n. 2, p. 52-63, maio/ago. 2003.

outras agências de fomento à pesquisa. Desta forma, qualificar nestes parâmetros os artigos de terapeutas ocupacionais brasileiros pareceu-nos fundamental para compreender, pelo menos em parte, a situação da pesquisa na área. Dos 145 artigos nacionais, 87 foram publicados em 24 revistas indexadas em bases nacionais e/ou internacionais, conforme Tabela 2 . Os demais 58 artigos foram publicados em 17 revistas brasileiras sem indexaçāo, sendo uma delas os Cadernos de Terapia Ocupacional da UFSCar.

A CAPES divide a classificação dos periódicos em nacional e internacional, sendo cada um deles com três níveis: Qualis internacional A, B e C; Qualis nacional A, B e C. Para a Grande Área da Saúde, a classificação de circulação internacional para Qualis A e B é feita a partir dos índices de impacto dos periódicos específicos de cada área. Estas são classificadas com base nas compilaçōes dos Citation Index do Journal Citation Reports (JCR) que é realizado pelo Institut for Scientific Infornation (ISI) ${ }^{(2)}$. No Qualis C internacional, estão os periódicos catalogados nas bases Medline, Intemational Pharmaceutical Abstracts (IPA), International Nursing Index (INT), Cumulative Index to Nursing \& Allied Health Literature (CINAHAL), SportDiscus, ERIC, Tropical Diseases Bulletin, Sociological Abstracts, Sociological Abstracts Linguistics and Languages Behavior, Planning/Policy \& Development $t^{3)}$.

No Qualis Nacional estão os periódicos, brasileiros ou não, que tenham corpo editorial, regularidade, consultores e periodicidade definida pelas áreas. O Qualis nacional A é composto pelos periódicos indexados no SciELO (Scientific Electronic Library
Online). No Qualis nacional B estão os periódicos, brasileiros ou não, indexados no LLACS, EMBASE, EXCERPTA MÉDICA, PSYCLIT ou editados por sociedades científicas representativas da área. No Qualis nacional $\mathrm{B}$, estão os periódicos com corpo editorial, regularidade, consultores e periodicidade. Prevê-se que sejam publicados por pesquisador pelo menos 3 artigos Qualis internacional A ou B a cada triênio.

Se estes parâmetros são discutíveis parà a produção brasileira, na Terapia Ocupacional, tornam-se evidentemente descolados de dados obtidos neste estudo e apresentados na Tabela 2 . A análise dos dados indicou uma concentração de publicações na base LILACS na ârea da saúde. Na educação e ciências sociais, a EDUBASE e Sociological Abstract são bases bibliográficas que indexam publicaçōes de artigos de terapeutas ocupacionais. Esta situação problemática foi patente, também, ná análise das publicaçôes estrangeiras.

Para nós, a discussāo deve favorecer o desenvolvimento a partir de parâmetros realistas e adequados para a produção de conhecimentos que respeitem as necessidades locais $\mathrm{e}$ incentive as revistas brasileiras da área.

Entre os 12 periódicos internacionais, 5 são indexados. Foram encontrados 24 artigos, sendo $10 \mathrm{em}$ língua inglesa, 8 espanhola, 4 francesa e 1 italiana. Apenas 9 artigos foram escritos por autores únicos (Tabela 3 ). As publicações internacionais representam uma pequena parcela da produção, revelando um diálogo prioritário entre os próprios profissionais ( $58 \%$ em revistas da área) e interno ao país $(85,7 \%$ em revistas brasileiras), conforme dados das Tabelas 1 e 4 respectivamente.

Tabela 3. Artigos de autores únicos e coletivos publicados em periódicos estrangeiros até 2000 , segundo instituições de ensino e indexadores das bases de dados americanas.

\begin{tabular}{|c|c|c|c|c|c|c|c|}
\hline \multirow{3}{*}{ Periódicos estrengeiros } & \multicolumn{5}{|c|}{ Artigos publicados até 2000} & \multirow{3}{*}{ Total } & \multirow{3}{*}{ Inctexadores } \\
\hline & \multirow{2}{*}{\begin{tabular}{|c|} 
De \\
autores \\
coletivas
\end{tabular}} & \multicolumn{4}{|c|}{ De autor único } & & \\
\hline & & USP & UFSCar & UFMG & PUCCantp & & \\
\hline 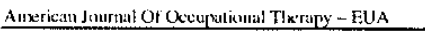 & 2 & & & & & 2 & Enbrase; Medline: Psychinliv; Lis; lakernational Nursing Inlex \\
\hline 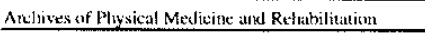 & 2 & & & & & 2 & Eubose: Medline: Isi; Jutenlationsal Nursing [ndex \\
\hline Occupationial Therarw Jounal of Reseurch - El:A* & 2 & & & & & 2 & Limbase \\
\hline Journal of Oukconk . Measurement & 1 & & & & & 1 & Modline \\
\hline Occupational Theruny International & 1 & 1 & & & & 2 & Nàn loni lex:alizands indexiader \\
\hline Diahruliz Rivistu Di Studi Itatici - Brasiz/ltália & 1 & & & & & 1 & 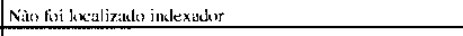 \\
\hline Ellwcathinal and Psychological Measurenxent - Calladú & 1 & & & & & 1 & PSYCHINFO \\
\hline Javernal D' Ergetherapie - França & 2 & 2 & & & & 4 & Näis foit hecalizados indexikbur \\
\hline Matćria Prinia - Argentinis & 2 & 2 & & & 1 & 5 & Nāes for loxalizado indexinkor \\
\hline Revista Aulnulorogies - Espanlsa & & & & & 1 & 1 & Nän, fori kxializado indexadur \\
\hline 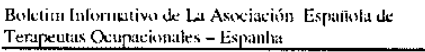 & 1 & & & & & 1 & Nìn fui kxualizado indexiadur \\
\hline Revista Toter Huspital - Espautha & & & & & 1 & 1 & 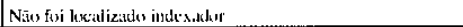 \\
\hline IOTAL & 15 & 5 & j** & $\therefore$ & 3 & 24 & \\
\hline
\end{tabular}

* a partir de 2002, o lítulo do periódico foi alterado para Occup̧ation, Particip̧ation and Heallh.

(2) Grande ârea da saúde: Educação Física, Enfermagem, Farmácia, Medicina I. Il e lll, Odontologia e Saúde Coletiva

(3) Para conhecer esses critérios consultar www.capes.gov.br 
BARROS, D. D., OLIVER, F. C. Contribuição para a discussão do Qualis de terapia ocupacional no Brasil.

Tabela 4. Artigos de autores únicos e coletivos publicados em periódicos nacionais de terapia ocupacional até 2000, segundo instituições de ensino e indexadores.

\begin{tabular}{|c|c|c|c|c|c|c|c|c|}
\hline \multirow{3}{*}{ Periódicos nacionais } & \multicolumn{6}{|c|}{ Artigos publicados até 2000} & \multirow{3}{*}{ Total } & \multirow{3}{*}{ Indexadores } \\
\hline & \multirow{2}{*}{$\begin{array}{l}\text { De autores } \\
\text { coletivos }\end{array}$} & \multicolumn{5}{|c|}{ De autor único } & & \\
\hline & & USP & UFSCar & UFMG & PUCCamp & UNISO & & \\
\hline Revista de Terapia Ocupacional da USP/SP & 15 & 27 & 4 & 1 & 1 & l & 49 & LILACS \\
\hline $\begin{array}{l}\text { Cadenos de Terapia Ocupacional da } \\
\text { UFSCar }\end{array}$ & 13 & 1 & 19 & - & 1 & l & 35 & Scm indexação \\
\hline Total & 28 & 28 & 23 & 1 & 2 & 2 & 84 & \\
\hline
\end{tabular}

Os 24 artigos internacionais foram publicados em 13 diferentes periódicos, destes pudemos identificar as bases de dados de 5 periódicos que reuniram 8 artigos de autores brasileiros. Esses dados demonstraram a dispersão dos artigos em grande número de revistas tanto nacionais como internacionais, evidenciando ausência de estratégia nacional para dar visibilidade ao crescimento acadêmico que efetivamente ocorreu no período (Tabela 3). Dos 145 artigos nacionais, 84 deles foram publicados numa das duas revistas especializadas, segundo demonstrado na Tabela 4. Observa-se que antes da edição desses periódicos, os artigos nacionais encontrados nos currículos pesquisados estavam dispersos em revistas da área da medicina e da psicologia e nesse período apenas 1 artigo internacional foi publicado no American Journal of Occupational Therapy (Tabela 3).

\section{O estrangulamento da produção pela falta da pós-graduação específica}

Com base nos dados apresentados, é possível levantar a hipótese de uma demanda crescente por formação de pós-graduação e além disso, considerar que grande parte das atividades de pesquisa no país está vinculada ao desenvolvimento de programa de pósgraduação. Assim, a consolidação da terapia ocupacional no Brasil é dependente de uma forte articulação entre ensino e pesquisa, o que atualmente, não pode prescindir de cursos de pós-graduação strictu sensi. No entanto, a criação desses programas tem enfrentado obstáculos importantes como os critérios de avaliação da atividade científica e acadêmica, as exigências para a abertura de novos programas e a insuficiente articulação dos próprios profissionais.

A criação de programas de estudos pósgraduados vem se modificando principalmente a partir de 1998 devido à implantação pela CAPES -
Coordenadoria Nacional de Aprimoramento do Ensino Superior - de novos processos de avaliação dos programas já existentes e exigências para criação de novos programas. Os programas são avaliados segundo a proposta do programa, que inclui a história de trabalho conjunto do grupo de pesquisa e infraestrutura; a composição do corpo docente que deverá contar com pelo menos $60 \%$ deles em regime de trabalho de 40 horas semanais, com produção bibliográfica compatível. Docentes de outras instituições poderão participar desde que não se caracterize dependência externa, ou seja, não sejam somente eles que possam atender aos requisitos mínimos de produção científica. Outro critério está na coerência entre a produção intelectual apresentada e as linhas e projetos de pesquisa. Também são utilizados critérios de avaliação e pontuação da produção bibliográfica dos docentes a partir do Sistema Qualis onde se privilegia (pontua-se de forma privilegiada) a produção de artigos em detrimento de livros, sendo melhor pontuados aqueles divulgados em periódicos participantes de bases de dados bibliográficos internacionais e em menor escala os artigos publicados em periódicos nacionais também credenciados em bases de dados.

Nesse sentido, os programas existentes passam a ser melhor considerados quando possuem docentes orientadores de suas próprias instituiçōes e departamentos. Dessa maneira, o credenciamento de terapeutas ocupacionais doutores em programas de outros departamentos e instituiçōes tornou-se praticamente inviável. Cabe ressaltar que esta vinha sendo a única estratégia disponível para dar continuidade à carreira acadêmica e para formar novos profissionais na área em estudos pós-graduados. Além de se privilegiar a vinculaçāo de orientadores, predominantemente da instituição proponente do programa, a aprovação de novos programas está baseada na avaliação da produção bibliográfica segundo critérios da área da saúde, incompatíveis com

'TPara maiores detalhes consultar www.capes gov.br 
as condições de desenvolvimento da terapia ocupacional. Assim, para a criação de programas de pós-graduação strictu sensu em terapia ocupacional, devem ser observados os mesmos critérios que para criação de cursos em farmácia, medicina, saúde coletiva, odontologia, educação física e enfermagem, que já têm programas de pós-graduação no país em fase de consolidação ou mesmo programas já tradicionais.

Sem possibilidade de inserção em programas de pós-graduação de áreas afins e com o enrijecimento dos critérios para criaçāo de novos programas, os terapeutas ocupacionais têm diante de si uma situaŗāo paradoxal: necessitam estar vinculados a programas de pós. graduação para construírem o percurso de pesquisadores, mas ao mesmo tempo, esse percurso só é possível se estiverem vinculados a algum programa já existente.

Não vivemos as mesmas condições universitárias das áreas que, hoje consolidadas, exigem e ditam os parâmetros de avaliação de novos programas. Elas próprias, quando de sua constituiçāo no final dos anos 70 e início dos anos 80 , não se submeteram aos critérios de avaliaçāo atualmente impostos. É preciso encontrar maneiras de superar os impasses através de iniciativas coletivas organizadas, torna-se fundamental conhecer por meio de diferentes perspectivas a produção da terapia ocupacional no país.

\section{Critérios de avaliação da produção intelectual na grande área da saúde}

O Sistema Qualis na Grande Área da Saúde organizou-se com base em diferentes critérios e velocidade em relação a outros campos do conhecimento. Seus parâmetros privilegiam aspectos da produção acadêmica, antagônicos aos de outras áreas do conhecimento e de pesquisa, tão consolidadas quanto às áreas da saúde, mas que pela natureza dos problemas e questões a que se dedicam admitem e privilegiam outras perspectivas de análise do contexto em que ocorre a produção bibliográfica. Nem todas as áreas de pesquisa qualificadas pela CAPES apresentaram seus parâmetros Qualis de forma quantitativa e classificatória, vide a área de Serviço Social, parte da grande área de Ciências Sociais aplicadas ou as áreas de Educação e de Psicologia ${ }^{(4)}$. Os parâmetros foram acordados por representantes de instituiçōes de pesquisa nas áreas e/cu por profissionais convocados para esse fim e, principalmente, a partir de processos de avaliaçãão de programas de pós-graduação já existentes, constituindorse parâmetros e processos de avaliação que buscaram representar as especificidades de cada área.

É fundamental que nos questionemos sobre quais seriam os principais aspectos a serem avaliados na produção intelectual em terapia ocupacional para sua consolidação como profissão e campo de pesquisa. Quando se estruturam parâmetros excessivamente rígidos de avaliação da produção acadêmica individual, condiciona-se a produção científica das áreas ao aumento do número de artigos publicados de forma a responder a necessidades de melhoria dos currículos dos pesquisadores, sem correspondente consolidação de grupos ou linhas de pesquisa.

A sobrevalorização da produção de artigos em periódicos internacionais em detrimento da produção de livros e de artigos nacionais, não possibilita diálogo com campos de pesquisa em constituição, onde a produção de obras gerais, de caráter formativo teriam importância estratégica, visto que é pequeno o número de títulos disponíveis no país, além de que a acessibilidade a livros é maior, assim como o hábito de leitura desse tipo de publicação. Sem discutir a especificidade das áreas esses critérios funcionam muito mais como processos de homogeneização da produção intelectual, e a esse respeito existem discussões colocadas.

Yarnamoto et al. (1999) são enfáticos em afirmar que embora não hạja

... uma identidade entre produção científica e publicação, os dois parâmetros utilizados na avaliação do desempenho cientílico de um campo têm se concentrado no número de artigos publicados $\mathrm{em}$ bases de dados de prestígio c o número de cilações que recebem. registrados nesses mesmos veículos (p. 2).

O parâmetro de avaliação para criação de cursos está indicado pelos índices de citação do Journal Citation Reports - JCR, que sāo índices bibliométricos de uso polêmico, uma vez que

os critérios de inclusão de periódicos adotados pelo ISI não são baseados na premissa de atingir uma cobertura representativa (no sentido estatístico) dit ciência produzida nos diversos países e regiões do mundo (COIMBRA Jr., 1999, p. 5).

Nessa avaliação utilizam-se indicadores elaborados a partir de metodologias da economia, estatística, documentação e administração, que derivam das compilações do Citation Index do Institute for Scientific Information - ISI - e de bases similares (SPINAK, 1998,p. 1).

Utilizar esse parâmetro como critério de avaliação tem sérias repercussões em diferentes campos do conhecimento, principalmente quando se observa a produção intelectual latino americana no cenário internacional. Em 1995, esta representava apenas 1,5\% do total de artigos indexados anualmente pelo ISI 
(GARFIELD apud SPINAK, 1998). O mesmo ccorre com a produção nacional, que conforme Meneghini (1998, p. 2) está representada

no $1 \mathrm{SI} \mathrm{em}$ apenas $20 \%$ ou $25 \%$ de scu total o que significa que $80 \%$ dela é pouco visível tanto para os pesquisadores brasileiros como estrangeiros". Para o autor: "essa parte majoritária da produção científica nacional não está imersa necessariamente por falta de qualidade. Ela está nessa circunstância por razōes diversas que não cabem aqui discutir. $\hat{E}$ importante, no entanto, dizer que existe interesse da comunidade científica internacional por muito do que está na base do iceberg.

Essa forma de avaliação da atividade acadêmica e intelectual não é internacionalmente consensual, como muitas vezes pode parecer, nem mesmo em nível nacional (COIMBRA Jr, 1999; FORATTINI, 1997, 1998; MENEGHINI, 1998). Métodos de avaliação alternativos têm sido propostos pela OCDE - Organização para a Cooperação e Desenvolvimento Econômico e pela UNESCO que publicou três diferentes manuais (Manual de Frascati, de Oslo e de Canberra) que tratan de oferecer subsídios para as pesquisas que buscam mensurar as atividades científicas, recursos humanos em Ciência e Tecnologia e as inovações tecnológicas. Desta forma, para a UNESCO as Atividades de Ciência e Tecnologia ACT - devem compreender o ensino e a formação científica e técnica, assim como os serviços científicos e lécnicos (SPINAK, i998, p. 6).

Também é bastante polêmica a inclusão de periódicos nas bases de dados internacionais. Historicamente elas não tinham intenção de avaliar a qualidade, mas procuravam incluir um maior número de itens sobre determinado campo. Posteriormente, com o aumento exponencial da produção bibliográfica os critérios de inclusão de periódicos nas chamadas bases tradicionais de indexação têm gerado acirradas discussões entre editores de periódicos, autores e técnicos de órgãos de fomento (COIMBRA Jr, 1999, p, 3).

As bases estrangeiras não têm por tarefa a inclusão das revistas publicadas no mundo, muito mais buscam responder às necessidades de arquivar, indexar e disponibilizar a literatura de seus países. Spinak (1998, p.5) ressaltal o caso da França e Inglaterra que desenvolveram importantes veículos de disponibilização da produção científica de seus países respectivamente através dos sistemas PASCAL e CAB. Além destes tanto) a China como a ex-Yugoslávia e a Bulgária criaram seus sistemas de informação e avaliação científica. As iniciativas como Litcratura Latinoamericana e do Caribe em Ciências da Saúde - LILACS e o Scicnific Electronic Library Online - SciELO - procuram trabalhar essa questão na América Latina e no Brasil.
Os estudiosos da sociologia da ciência atribuem importância relativa a esse tipo de avaliação, segundo a utilização de critérios intrínsecos ou extrínsecos. Jsto é, os primeiros enfatizam a lógica dos descobrimentos e as demandas intrínsecas ao próprio campo e os segundos defendem a importância de se perceber a atividade científica no contex to sociocultural, político e econômico no qual está enraizada. Neste caso, a ciência e, consequientemente, a produção científica não poderiam ser avaliadas em uma escala absoluta.

A chamada ciência internacional (o mainstream) é, $\mathrm{em}$ grande medida, o resultado das 'ciências nacionais' e produto da intertclação entre os fatores intrínsecos e extrínsecos (SPINAK, 1998, p. 6)

\section{Bases de dados bibliográficas internacionais e as necessidades da produção intelectual em terapia ocupacional no Brasil}

Uma das dificuldades a ser superada pelos pesquisadores brasileiros é a hipótese de um caráter universal intrínseco ao trabalho científico, sendo considerados subdesenvolvidos ou de caráter apenas local, os trabalhos e os cientistas que, comprometidos com as necessidades de países do terceiro mundo, encontram maior dificuldade em divulgar suas pesquisas em veículos de prestígio, frequientemente, pertencentes aos centros produtores de conhecimento e de acumulaçāo financeira do primeiro mundo (FORATTINI, 1996b, 1997, p. 1). Se o conhecimento deve manter seu caráter universal, o interesse na disseminação de resultados de estudos realizados sobre problemáticas relevantes para as populações da América Latina, da África ou da Índia é sempre menor para os editores dos veículos de primeiro mundo (FORATTINI, 1996b, p. 1-5).

No que se refere à terapia ocupacional, a situação é semelhante, mas com algumas contradições específicas. A pressa e a superficialidade de avaliação podem levar a erros enormes. Acreditamos que é preciso analisar os inúmeros aspectos e considerar diferentes perspectivas antes de definir uma estratégia para a terapia ocupacional. O pragmatismo excessivo pode não ser, neste momento, adequado a médio e longo prazo. $\mathrm{O}$ que parece óbvio deve ser posto à prova da investigação e da análise.

Como o campo da pesquisa em terapia ocupacional está em constituição é importante que lambém se possa refletir sobre a autonomia que se deseja construir, de que maneira se pretende estabelecer um diálogo com pesquisadores e centros de excelência estrangeiros. O ISI é base que leva à classificação da 
produção nela indexada como Qualis. Seria esta uma compreensão indiscutîvel para a terapia ocupacional'? Será que o desenvolvimento da terapia ocupacional tem parâmetros internacionais consolidados para serem aplicados sem questionamentos?

Entre os periódicos de terapia ocupacional, apenas o American Joumal of Occupational Therapy, está indexado no ISI. Este periódico está voltado para as questões nacionais, publicando prioritariamente trabalhos de profissionais e pesquisadores de aproximadamente 200 instituições de ensino norteamericanas com graduação na área. A única revista internacional com preocupação de veicular a produção de terapia ocupacional de diferentes paises é a Occupational Therapy Intermational que não está indexada naquela base de dados. Existem, também, iniciativas de criação de bases de dados específicas como OT Sys or Bib, localizadas no decorrer deste trabalho. Existe acentuada hierarquia entre as bases, valorização maior dos periódicos indexados em bases de dados bibliográficos da área médica de línguas anglosaxônicas e de bases organizadas por instituiçōes norte americanas, em detrimento de bases européias.

O privilégio às bases de dados e periódicos internacionais colocados a priori nāo leva em conta a natureza e a importância dos problemas que vêm sendo tratados, nem sempre de interesse para a comunidade científica internacional. Ao mesmo tempo, a qualidade da produção científica depende também da posição que ocupamos como pesquisadores, ou seja da interlocução que é produzida. A esse respeito é importante conhecer as reflexões de Xavier et al. (2003) sobre o número de publicações brasileiras em revistas de circulação internacional, indagando se esses números refletem independência intelectual ou se

refletem dependência em relação aos laboratórios internacionais, cabendo aos laboratórios nacionais o papel subalterno de fornecimento de espécimes ou amostras de material de pesquisa e/ou de coleta de dados, sem cilvolvirnento nas etapas de planejamento, geração de hipóteses, interprctação final dos resultados e mesmo de redação do artigo para publicação (p. 46).

Apesar das publicações conjuntas de pesquisadores de instituiçōes diferentes e de diferentes países poderem ser resultado de processo de colaboração científica, há o risco de que sejam formas de facilitar a aceitação de artigos em periódicos internacionais sem corresponder efetivamente a uma atividade de intercâmbio científico com vitalidade $\mathrm{e}$ independência. O que, entretanto, preocupa é a valorização de publicações em periódicos estrangeiros em detrimento do valor, que também existe, de artigos engajados na constituição e fortalecimento do pensamento crítico e prcocupados com questões brasileiras relevantes, nem sempre de interesse estrangeiro compatível.

No caso da terapia ocupacional, observou-se que dos 24 artigos publicados em periódicos estrangeiros, 6 foram publicados em revistas norteamericanas por autor brasileiro com autores daquele país. Já nos artigos publicados em revistas canadense, britânica, francesa, espanhola ou italiana os autores brasileiros publicaram sozinhos ou em grupo.

A terapia ocupacional brasileira tem tido um desenvolvimento diferenciado segundo as regiões do país em que se constituiu ensino e pesquisa específico. Entre estas diferenças, chamou-nos a atenção que exista um diálogo preferencial com a Europa por parte das instituições paulistas (PUCCampinas, UFSCar e USP) enquanto que em Minas Gerais, esse intercâmbio tem sido feito preferencialmente com a América do Norte. Tais diferenças revelaram-se, também, nos estudos pós-graduados: mestrados, doutorados e nos pósdoutorados. Alguns profissionais buscaram pós-graduação específica em instituiçōes norteamericana, canadense e inglesa principalmente, facilitando parcialmente o início diálogo entre profissionais brasileiros e estrangeiros. Outros preferiram realizar a formação no Brasil em áreas afins e construíram intercâmbios com instituições de ensino e pesquisa tanto das ciências da saúde como das ciências humanas pertinentes ao campo de pesquisa a que se dedicaram e, ainda, com serviços assistenciais de referência internacionais.

Deste percurso, resultaram colaborações e intercâmbios profissionais sob a forma de estágios, participação em congressos, assessorias, pesquisas conjuntas e publicações. Apesar da importância, os intercâmbios são ainda numericamente pequenos e nāo têm evoluído para cooperações institucionais, em grande parte, pela dificuldade de inserção na pós-graduação de terapeutas ocupacionais.

No Brasil, o esforço de divulgação do conhecimento produzido em terapia ocupacional tem sido uma constante para os dois únicos periódicos existentes: Cadernos de Terapia Ocupacional da UFSCar e Revista de Terapia Ocupacional da USP. Essas iniciativas, aliadas às de diferentes autores nacionais que têm publicado livros sobre sua experiência profissional ou suas pesquisas de mestrado ou doutorado, têm sido fundamentais na promoção do intercâmbio no país e na América Latina. Essas iniciativas se mantêm, apesar dos escassos apoios e das dificuldades conhecidas para a manutenção de revistas científicas e edição de livros com circulação 
significativa no país, sobretudo em campos como a terapia ocupacional, em que as Universidades não têm oferecido condições para o desenvolvimento e ampliação das atividades acadêmicas como observado pela inserção institucional de diferentes cursos.

A terapia ocupacional não conseguiu, até o presente, ser reconhecida como área específica e emergente com os incentivos correspondentes, necessários para sua consolidação. A capacitação docente e de pesquisadores tem sido resultado de esforços individuais e não de estratégias ou diretrizes institucionais.

Neste estudo, ficaram evidentes os limites de uma avaliaçāo da produção docente restrita àquela indexada em bases de dados bibliográficas, sem considerar a história recente do ensino de graduação, da formaçāo de pesquisadores e do próprio campo profissional no Brasil. As instituições de cnsino - desde a regulamentaçāo da profissão em 1969 - têm centrado suas preocupações na estruturaçāo da graduação, prejudicando enormemente a produção do conhecimento na área em todo país o que tem penalizado individualmente os pesquisadores. Estes têm sido avaliados tanto por suas instituições de ensino como pclos órgãos de fomento à pesquisa, sem considerar o fato de ser uma área emergente e em processo de estruturação. Nestas avaliações a produção acadêmica é analisada de forma descontextualizada c a partir de critérios definidos para as áreas consolidadas da saúde. Nesse sentido, será igualmente necessário pôr em questão a pertinência da classificação adotada atualmente pelo $\mathrm{CNPq}$ (e outros órgãos de fomento à pesquisa) onde a terapia ocupacional permanece vinculada à fisioterapia, área cuja afinidade é reconhecida, mas também bastante limitada, pois não acompanha as diferentes dimensōes ligadas ao campo das humanidades e das artes. Essa questão vem sendo colocada para o $\mathrm{CNPq}$, que tem se mostrado sensível à mudança solicitada.

Por outro lado, é fundamental investir institucionalmente na discussāo dos atuais critérios para criação de programas de pós-graduação na área e construir caminhos possíveis para a terapia ocupacional como campo de pesquisa. Aceitar individualmente os parâmetros colocados pelos processos de avaliação da produção intelectual pode parecer suficiente ou necessário, no entanto, não admite e trata da complexidade do trabalho acadêmico. É necessário fomentar o debate coletivo entre pesquisadores e docentes da terapia ocupacional brasileira para apostar criativamente em estratégias para lidar com os desafios que aqui em parte caracterizamos.

Agradecemos a colaboração de Aline Gomes Medina. Amanda Baron e Tânia Canhadas, alunas Je graduação que realizaram levantamento de clados deste estudo è a Erica Gasparolto Venturini pelos gráficos c revisão do texto.

BARROS, D.D., OLIVER, F.C. Contributing for the discussion of the Qualis classification in occupational therapy in Brazil. Rev. Ter. Ocup. Univ. São Paulo, v. 14, n. 2, p. 52-63, maio/ago 2003.

\begin{abstract}
Objective: To characterize the bibliographic production of occupational therapy researchers who belong to research groups coordinated by occupational therapists affiliated with the National Council for Scientific and Technological Development -- CNPq (Directory of Research Groups, version 4.1). The latter account for most researchers directly involved in rescarch activitics in the country. Methodology: Complete articles written by master's and/or doctoral occupational therapists, published in national or international journals until the year 2000 , were considered. These articles were researched at the $\mathrm{CV}$ database of $\mathrm{CNPq}$. Bibliographic production was characterized according to journals (indexed or not in bibliographic databascs), areas of knowledge (biology, health, education, social studies) and author's instilution of origin. Results: A total of 169 articles written by 46 authors were identified. These aticles were written from 1985 to 2000 , and published in 54 journals. National production accounted for $85 \%$ of the 169 articles. Master's and doctorate courses taken by occupational therapists had an impact on the bibliographic production only in the 1990s. In occupational therapy, an emerging field, the following was observed: authors are mainly distributed in the southeast, cspecially in the State of São Paulo; publication of articles is spread over various journals; there is a limited number of authors and production is low in absolute number. It was noted that the evaluation of bibliographic production of professors according to Qualis (classification of pcriodicals, joumals, proceedings and magazines) overestimates bibliographic production indexed in English bibliographic databases. The need tv establish more collective strategies to increase bibliographic production - of books and of articles - in absolute number was observed, as well as the need to urge discussion on the subject among researchers and professors.
\end{abstract}

KEY WORDS: Occupational therapy/education. Education. Research. Strategies. 


\section{REFERÊNCIAS}

ANTONIO, I. ; PACKER, A. Seminário sobre avaliação da produção científica: relatório final. Ci. Inf. [orline], v. 27. n. 2, 1998. Disponível em: <http://www.sciclo.br.>.

BARROS, F. A. de. Os descquilíbrios regionais da produção técnico-científica. São Paulo Perspectiva [onljne], v.14, n.3, 2000. Disponivel em: <http://www.scielo.br>.

BRISOLLA, S. N. Indicadores para apoio à tomada de decisão. Ci. Inf. [online], v. 27, n. 2, 1998. Disponível em: <hitp:// www.sciclo.br>.

COIMBRA Jr. C. E. A. Produção científica em saúde pública c as bases bibliográficas internacionais. Cad. Saúde Pública [online], v. 15, n. 4, 1999. Disponível em: <http:// www.sciclo.br>.

COIMBRA Jr, C. E. A. Desafios da produção e da comunicação científica em saúde no Brasil. Cad. Saúde Pública [online], v. 19, n.1, 2003. Disponível em: <http:// www.scielo.br>

COIMBRA Jr, C. E. A. Quem, onde, quando, o quê e o porquê. Rev. Saúde Pública [onlinc], v. 30, n .6, 1996 b. Disponivel em: <http://www.scielo.br>.

COIMBRA Jr, C. E. A. A intencionalidade da ciència. Rev. Saúde Pública [online], v. 31, n. 2, 1997. Disponível em: <http://www.scielo.br>.

COIMBRA Jr, C. E. A. A ciência e a sociedade. Rev. Saúde Pública [on line], v. 33, n. 1, 1999. Disponível em: <http:// www.scielo.br>

COIMBRA Jr, C. E. A. A comunidade européia, o curo c a ciência. Rev. Saúde Pública [onlinc], v. 33, n. 3, 1999. Disponivel em: <lutp://www.sciclo.br>.

COIMBRA Jr, C. E. A. A privatização da universidade c o genoma. Rev. Saúde Pública [online], v. 35, n. 2, 2001. Disponivel cm: <http://www.scielo.br>.

EMMEL, M. L. G.; LANCMAN, S. Quem são nossos mestres e doutores? O avanço da capacitação docente $\mathrm{cm}$ terapia ocupacional no Brasil. Cad. Ter. Ocup. UFSCar. v. 7. n. 1, p. 29-38, 1998.

FORATTINI, O. P. A tríade da publicação cientílica. Rev. Saúde Pública [online], v. 30, n. 1, 1996a. Disponível em: <http:/www.scielo.br>.

GAMBOA, J. O. A. Accso a revistas latinoamericanas en Internet. Una opción a través de las bases de datos clase y periódica. Ci. Inf. [online], v. 27, n. 1. 1998. Disponível em: <http://www.scielo.br>.

LANCMAN, S. A influência da capacitação dos terapeutas ocupacionais no processo de constituição da profissão no Brasil. Cad. Ter. Ocup. UFSCar, v. 7, n. 2, p. 49.57, 1998.

LOPES, R. E. A formação do terapeuta ocupacional. O currículo: histórico e propostas alternativas. 1991. Dissertação (Mestrado) - Centro de Educação e Ciências Humanas. Universidade Federal de São Carlos, São Carlos, 1991.

MAGALHÃES, L. Situação da capacitação docente c perspectivas de criação de cursos de pós-graduação em terapia ocupacional na Universidade Federal de Minas Gerais, UFMG. Rev. Ter. Ocup. Univ. São Paulo, v. 9. n. 3, p. 119-24. 1998.

MÂNGIA, E. F. Terapia ocupacional: práticas, discursos e a questão da legitimidade científica. Rev. 'Ter. Ocup. Univ. São Paulo, v. 10, n. 2/3, p. 36-41, mai./dez., 1999.

MENEGIIINI, R. Avaliação de produção científica e o projeto SciELO. Ci. Inf.[online], v. 27, n. 2, 1998. Disponivel em: <http://www.scielo.br>.

PRAT, A. M. Avaliação da produção científica como instrumento para o desenvolvimento da ciência e da tecnologia. Ci. Inf. [online], v. 27, n. 2, 1998. Disponivel em: <http:// www.sciclo.br>.

SPINAK, E. Indicadores cienciométricos. Ci. Inf. [online]. v. 27, n. 2. 1998. Disponível em: <http://www.scielo.br>.

YAMAMOTO, O. H.; SOLZA, C.C. de; YAMAMOTO. M. E. A Produção cientílica na psicologia: uma análise dos periódicos brasileiros, período 1990-1997. Psicol. Reflex. Crit., Porto Alegre, v. 12, n. 2, p. 1-15, 1999.

YAMAMOTO, O. H.; MENANDRO, P. M.; KOLLER, S H.; LOBLANCO, A. C.; HUTZ, C. S.; BUENO, J. L. GUEDES, M. C. Avaliação de periódicos científicos brasileiros da área da psicologia. Ci. Inf. lonline], v. 31, n. 2, 2002. Disponivel em: <http://www.scielo.br>.

MIRANDA, A. Produção científica na ciência da informação. Ci. Inf. [online], v. 27, n. 1, 1998. Disponível em: <http:// www.scielo.br>. Acesso em: 2 jul. 2003.

NORONHA, D. P. Análise das cilações das dissertações de mestrado e teses de doutorado em saúde pública (19901994): cstudo exploratório. Ci. Inf. [online]. v. 27, n.l. 1998. Disponivel cm: <http://www.scielo.br. Acesso cm: 2 jul. 2003.

Recebido para publicaçũo: Fevereiro de 2003

Accito para publicação: Março de 2003 